\title{
SPHERES IN $E^{3}$ WHICH ARE HOMOGENEOUS OVER A 0-DIMENSIONAL SET
}

\author{
C. E. BURGESS
}

\begin{abstract}
A 2-sphere $S$ in Euclidean 3-space $E^{3}$ is defined to be homogeneous over the subset $X$ of $S$ if for each $p, q \in X$ there is a homeomorphism $h: E^{3} \rightarrow E^{3}$ such that $h(S)=S$ and $h(p)=q$. It is shown that a 2-sphere $S$ in $E^{3}$ is tame from one side provided $S$ is locally tame modulo a tame 0 -dimensional set $C$ such that $S$ is homogeneous over $C$. An example is described to show that it is necessary to require that $C$ be tame.
\end{abstract}

It is not known whether a 2-sphere $S$ is tame in $E^{3}$ if $S$ is homogeneous in $E^{3}$; i.e., for each $p, q \in S$ there is a homeomorphism $h: E^{3} \rightarrow E^{3}$ such that $h(S)=S$ and $h(p)=q[4$, Question 4.5.3], [5, p. 312]. In this paper we show that a 2 -sphere $S$ in $E^{3}$ is tame from one side provided $S$ is locally tame modulo a tame 0-dimensional set $C$ such that $S$ is homogeneous over $C$. ( $S$ is defined to be homogeneous over the subset $X$ of $S$ if for each $p, q \in X$ there is a homeomorphism $h: E^{3} \rightarrow E^{3}$ such that $h(S)=S$ and $h(p)=q$.) Our proof of this theorem mainly involves showing that $S$ can be locally spanned from one side [3, Theorem 7]. This theorem could be considered an extension of the theorem by Harrold and Moise [6] that a 2-sphere $S$ in $E^{3}$ is tame from one side if $S$ is locally tame modulo one point. We indicate that the Alexander horned sphere [1] is homogeneous over the tame Cantor set where the sphere is wild. We then notice, with an example described by Bing [2], that it is necessary to require, in the hypothesis of Theorem 1, that the 0-dimensional set $C$ be tame in $E^{3}$.

We define a 2-sphere $S$ in $E^{3}$ to be tame from one side if either $S \cup \operatorname{Int} S$ is a 3-cell or $S \cup$ Ext $S$ is homeomorphic to the closure of the complement of a round ball in $E^{3}$. A subset $X$ of a 2 -sphere $S$ in $E^{3}$ is defined to be tame if $X$ is a subset of some tame sphere in $E^{3}$. If $S$ is a 2-sphere in $E^{3}, U$ is a component of $E^{3}-S$, and $x \in S$, we say that $S$ can be locally spanned at $x$ from $U$ provided that for each $\varepsilon>0$ there exist disks $D$ and $D^{\prime}$ such that:

(1) $x \in \operatorname{Int} D \subset S$;

(2) $\operatorname{Bd} D=\operatorname{Bd} D^{\prime}$;

(3) Int $D^{\prime} \subset U$;

(4) $\operatorname{Diam}\left(D \cup D^{\prime}\right)<\varepsilon$.

Received by the editors July $21,1975$.

AMS (MOS) subject classifications (1970). Primary 57A10, 57A35, 57A45, 57A50.

Key words and phrases.Tame spheres, homogeneous embeddings, horned spheres. 
We rely on [5] for various other definitions and notation.

THEOREM 1. If $S$ is a 2-sphere in $E^{3}$ and $C$ is a 0 -dimensional tame subset of $S$ such that $S$ is homogeneous over $C$ and $S$ is locally tame at each point of $S-C$, then $S$ is tame from one side.

Proof. We will show that there is a component $U$ of $E^{3}-S$ such that $S$ can be locally spanned from $U$ at each point of $S$, and this will imply that $S$ is tame from $U$ [3, Theorem 7]. We consider $S$ to be locally wild at each point of $C$, for otherwise the hypothesis would imply that $S$ is locally tame. Since $S$ is locally tame at each point of $S-C$, we may assume that $S-C$ is locally polyhedral [5, Theorem 4.4.5]. Let $\varepsilon$ be a positive number and $D_{0}$ a disk in $S$ such that $C \cap \operatorname{Int} D_{0} \neq 0$ and $\operatorname{Diam} D_{0}<\varepsilon / 2$. Since $C$ is tame, there exists a polyhedral sphere $S_{1}$ which has diameter less than $\varepsilon / 2$ and encloses a point of $C \cap \operatorname{Int} D_{0}$ such that $S \cap S_{1} \subset \operatorname{Int} D_{0}$ and $S_{1} \cap C=0$. We consider $S$ and $S_{1}$ to be in relative general position so that each component of $S \cap S_{1}$ is a simple closed curve. By standard cut-and-paste techniques [5, p. 262], we adjust $S_{1}$ by removing any component of $S \cap S_{1}$ which is the boundary of a disk in Int $D_{0}-C$. With such an adjusted $S_{1}$, we obtain disks $D_{1}$ and $E_{1}$ such that:

(1) $E_{1} \cap S=\mathrm{Bd} D_{1}=\mathrm{Bd} E_{1}$;

(2) $D_{1} \subset \operatorname{Int} D_{0}$ and $E_{1} \subset S_{1}$;

(3) $\operatorname{Diam}\left(D_{1} \cup E_{1}\right)<\varepsilon$;

(4) $C \cap \operatorname{Int} D_{1} \neq 0$.

An iteration of this procedure enables us to obtain a null sequence $\left\{S_{i}\right\}$ of 2-spheres and null sequences $\left\{D_{i}\right\}$ and $\left\{E_{i}\right\}$ of disks such that for each $i$, requirements (1)-(4) are satisfied with $S_{1}, D_{1}$, and $E_{1}$ replaced with $S_{i}, D_{i}$, and $E_{i}$, respectively, and with $D_{0}$ replaced with $D_{i-1}$. Let $p=\cap_{i=1}^{\infty} D_{i}$. There is a component $U$ of $E^{3}-S$ such that infinitely many of the Int $E_{i}$ 's are in $U$. Thus $S$ can be locally spanned at $p$ from $U$. The hypothesis that $S$ is homogeneous over $C$ implies that $S$ can be locally spanned from $U$ at each point of $C$. With $S$ locally tame at each point of $S-C$, we conclude that $S$ is tame from $U[3$, Theorem 7].

THEOREM 2. The Alexander horned sphere $S$ is homogeneous over the Cantor set $C$ of points where $S$ is wild.

INDICATION OF PROOF. In Figure 1, we indicate an Alexander horned sphere $S$ which is tame from Ext $S$. We follow Bing's description in [2] where the $D$ 's are disks, the $T$ 's are tubes, and the $K$ 's are solid cylinders. A point $x$ is in the Cantor set $C$ of points where $S$ is wild if and only if $x=K_{n_{1}} \cap K_{n_{1} n_{2}} \cap K_{n_{1} n_{2} n_{3}}$ $\cap \ldots$ and, for each $i, n_{i}$ is either 1 or 2 . Let $p$ and $q$ be two points of $C$. It is our purpose to show that there is a homeomorphism $h: E^{3} \rightarrow E^{3}$ such that $h(S)=S$ and $h(p)=q$. We do this by identifying a sequence $\left\{h_{i}\right\}$ of homeomorphisms of $E^{3}$ onto itself which converges to the desired homeomorphism $h$. For convenience, we assume that $q=K_{2} \cap K_{22} \cap K_{222} \cap$. . . If 
$p \in K_{2}$, let $h_{1}$ be the identity homeomorphism on $E^{3}$. If $p \in K_{1}$, let $h_{1}$ be a homeomorphism of $E^{3}$ onto itself which revolves $E^{3} 180^{\circ}$ about an appropriate horizontal line such that $h_{1}(S)=S$ and $h_{1}\left(K_{1}\right)=K_{2}$. If $h_{1}(p) \in$ $K_{22}$, let $h_{2}$ be the identity homeomorphism on $E^{3}$. If $h_{1}(p) \in K_{21}$, let $h_{2}$ be a homeomorphism of $E^{3}$ onto itself which is the identity except near $K$ and which rotates both $D_{1}$ and $D_{2} 180^{\circ}$ in the same direction such that $h_{2}\left(T_{21}\right)=$ $T_{22}, h_{2}\left(K_{21}\right)=K_{22}$, and $h_{2}(S)=S$. Similarly, $h_{3}$ is either the identity homeomorphism on $E^{3}$ or a homeomorphism $h_{3}: E^{3} \rightarrow E^{3}$ which is the identity except near $K_{2}$ such that $h_{3}\left(K_{221}\right)=K_{222}$ and $h_{3}(S)=S$. This process can be continued to obtain a sequence $\left\{h_{i}\right\}$ of homeomorphisms which converges to the desired homeomorphism $h$.

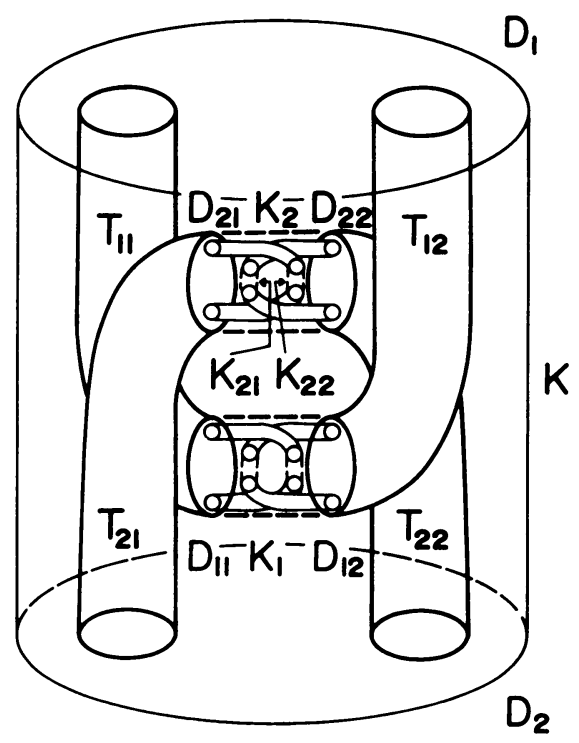

FiguRE 1

REMARK 1 . It is necessary to require in Theorem 1 that the 0 -dimensional set $C$ be tame in $E^{3}$. The proof indicated above for Theorem 2 shows that for each $p, q \in C$, there is a homeomorphism $h$ of $S \cup$ Int $S$ onto itself such that $h(p)=q$. Furthermore, the homeomorphism $h$ could be constructed such that, for some $t \in \operatorname{Int} S, h(t)=t$. Bing [2] showed that $S^{3}$ results from sewing $S \cup$ Int $S$ to itself with the identity on $S$. Thus, by omitting the point $t$ in one copy of $S \cup \operatorname{Int} S$, we have a 2 -sphere $S$ in $E^{3}$ such that (1) $S$ is locally tame modulo a wild Cantor set $C$, (2) $S$ is wild from each side at each point of $C$, and (3) $S$ is homogeneous over $C$.

REMARK 2 . We can adapt Theorem 1 to $S^{3}$ by requiring that the homeomorphism $h$, identified in the definition that $S$ is homogeneous over the Cantor set $C$, should not interchange the components of $S^{3}-S$. Without this additional requirement, we could change the construction indicated in Figure 1 such that the Cantor set $C$, of points where $S$ is wild, is the union of 
two disjoint tame Cantor sets $C_{1}$ and $C_{2}$ with $S$ locally tame from Int $S$ at each point of $C_{1}$ and locally tame from Ext $S$ at each point of $C_{2}$. This construction can be done so that $S$ is homogeneous over $C$.

\section{REFERENCES}

1. J. W. Alexander, An example of a simply connected surface bounding a region which is not simply connected, Proc. Nat. Acad. Sci. U.S.A. 10 (1924), 8-10.

2. R. H. Bing, A homeomorphism between the 3-sphere and the sum of two solid horned spheres, Ann. of Math. (2) 56 (1952), 354-362. MR 14, 192.

3. C. E. Burgess, Characterizations of tame surfaces in $E^{3}$, Trans. Amer. Math. Soc. 114 (1965), 80-97. MR 31 \#728.

4. __ Embeddings of surfaces in Euclidean three-space, Bull. Amer. Math. Soc. 81 (1975), 795-818. MR 51 \# 11514.

5. C. E. Burgess and J. W. Cannon, Embeddings of surfaces in $E^{3}$, Rocky Mountain J. Math. 1 (1971), 259-344. MR 43 \#4008.

6. O. G. Harrold, Jr. and E. E. Moise, Almost locally polyhedral spheres, Ann. of Math. (2) 57 (1953), 575-578. MR 14 \# 784.

Department of Mathematics, University of Utah, Salt Lake City, Utah 84112 